\title{
Des-patriarcalizar y Des-colonizar la Educación. Experiencias para una Formación Feminista del Profesorado
}

\author{
Non-patriarchal and Non-colonial Education. Experiences of a \\ Feminist Teacher Training
}

\author{
Irene Martínez Martín* \\ Gema Ramírez Artiaga
}

Universidad Complutense de Madrid

\begin{abstract}
Este artículo busca incluir una perspectiva feminista en la formación inicial del profesorado como respuesta a la necesidad de subvertir la reproducción de las desigualdades de género en el ejercicio de la docencia. Se toma como referencia una metodología de enfoque cualitativo y emancipador con carácter analítico-crítico fundamentada en la práctica de la investigación-acción-participativa dividida en las siguientes fases: a) estudio documental y bibliográfico en torno a narrativas contrahegemónicas feministas; b) construcción de una pedagogía feminista postcolonial para la formación del profesorado; y c) puesta en práctica de un proyecto piloto de formación feminista del profesorado y su posterior evaluación. Como principales resultados el artículo recoge: 1) la necesidad de posicionarse en un plano de pensamiento y acción feminista postcolonial ante la reproducción de desigualdades de género a través del ejercicio de la docencia; 2) la propuesta de construcción de una pedagogía feminista en la formación del profesorado bajo los principios de transformación y crítica social para des-colonizar y des-patriarcalizar saberes y prácticas educativas; y 3) la puesta en práctica de una experiencia piloto en la formación inicial del profesorado para trabajar temas de género e igualdad.
\end{abstract}

Descriptores: Educación, Coeducación, Formación de docentes, Movimiento de liberación femenina, Diversidad cultural.

\begin{abstract}
This paper wants to include a feminist approach in the initial teachers training as an answer to the reproduction of gender inequalities in the education. It takes as a methodology the qualitative and emancipatory approach with a point of view analytical and critical. All of this is based on the practice of research-action which is divided into the following phases: A) documentary and bibliographic study about non-hegemonic and feminist narratives; B) to make a postcolonial feminist pedagogy for teacher training; And c) implementation the feminist teachers training and their evaluation. As main results the paper includes: 1) the need to think and to live our lives in a postcolonial feminist approach with the goal to avoid the reproduction of gender inequalities in the education; 2) a feminist pedagogy wants to start a social changes, as the teacher training that is very important to decolonize and de-patriarchalize the knowledge and the educational practices; and 3) The implementation of a feminist teacher training as an experience to change the traditional education on gender and equality issues.
\end{abstract}

Keyswords: Education, Coeducation, Teacher education, Women liberation movement, Cultural diversity.

*Contacto:imartio2@ucm.es

ISSN: 2254-3139

www.rinace.net/riejs/

revistas.uam.es/riejs
Recibido: $\quad 15$ de abril 2017

$1^{\text {a }}$ Evaluación: 23 de junio 2017

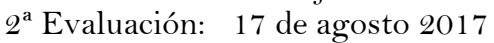

Aceptado: $\quad 26$ de septiembre 2017 
Creo que ésta será una lucha infinita (la feminista) y que las victorias que conquistamos nos permiten imaginar nuevas libertades. Creo que cada generación va a crear nuevos significados sobre lo que significa ser libre.

(Angela Davis, 1944)

\section{Introducción: La importancia de la socialización del género hegemónico y la reproducción de desigualdades}

Ante la reproducción de los mecanismos de desigualdad patriarcales las teóricas feministas (Amorós, 2007; Lagarde, 2012; Segato, 2016) ponen el acento en la socialización machista y sexista presente tanto en esferas privadas como en esferas públicas. Los agentes de socialización (familias, instituciones educativas, medios de comunicación, etc.) crean y transmiten códigos culturales que sustentan un sistema organizado en estructuras opresoras y que asumimos como legítimas y propias.

Los valores, conductas e imaginarios son elementos aprendidos que, a su vez, se transmiten mediante los diferentes mecanismos de socialización y se reproducen a través de esos códigos culturales hegemónicos. Hablamos de unos mandatos de género normativos -masculinos y femeninos- aprendidos y que nos construyen como mujeres u hombres en términos binarios de exclusión (Varela, 2008).

Segato (2016) señala que las relaciones entre los géneros se organizan en términos de jerarquización donde lo masculino está en una posición de poder sobre lo femenino. Ya Beauvoir (1981) puso el acento en el hecho de que no se nace mujer, sino que se llega a serlo. A esta construcción social de los géneros normativos se van asociando una serie de conductas, características y dimensiones consideradas como normales y definitorias de la masculinidad y la feminidad dominante. En este sentido, un hombre normativo es definido por su fuerza, su posibilidad de proveer a partir de un trabajo remunerado y considerado como productivo, entre otras. Por el contrario, una mujer normativa es definida por su capacidad de cuidado y atención a su familia, por ser emocional y delicada y por buscar al hombre con el que formar una familia nuclear basada en los principios del amor romántico y heteronormativo.

Partiendo de estas evidencias, en este artículo fijamos nuestra mirada en la importancia que tiene la socialización de tales mandatos de género en la construcción de la masculinidad y feminidad hetero-designada (Butler, 2007) y hegemónica basados en una lógica de poder. Como respuesta ante las desigualdades que supone entender el género de este modo (construcción binaria y jerarquizada), proponemos la deconstrucción de las categorías hegemónicas de: "hombre-mujer"- "masculino-femenino" y los mandatos de género asociados. Para ello ponemos el foco en la educación tradicional que reprime una humanidad diversa, donde la masculinidad y la feminidad son definidas bajo unos mandatos rígidos y donde esa feminidad se pone al servicio de los privilegios de dicha masculinidad. Ngozi (2015) en su famoso discurso "todos/as deberíamos ser feministas" señala cómo:

Las niñas aprenden a encogerse, a hacerse pequeñas, a esperar un hombre salvador, a cuidar y a ser emocionales. Las mujeres podemos tener ambición, pero no demasiada; tener éxito, pero no demasiado (...) Educamos a las mujeres a ser competidoras por el amor de los hombres. No por puestos de trabajo o logros personales. Enseñamos a las chicas que no pueden ser seres sexuales de la misma manera que los chicos. A ellas las elogiamos por su maternidad, a ellos no. 
Enseñamos a las chicas a tener vergüenza. A sentir culpa sólo por haber nacido mujeres. Mujeres que crecen silenciándose de si mismas. Que no pueden sentir deseo o decir lo que piensan. (Ngozi, 2015, p. 41)

Las teóricas feministas citadas hasta el momento se hacen eco de cuán naturalizados están estos estereotipos y mandatos de género lo que dificulta que nos podamos percatar de su origen: constructo social y cultural. Poniendo el acento en la socialización hegemónica observamos cómo las identidades genéricas se construyen por oposición (Connell, 1997) y se completan con un variado "deber ser" (proveedor, protector, cuidadora, emocional...). A esta construcción normativa de las identidades genéricas se suma la intersección de otras dimensiones (Mohanty, 2008), tales como la raza, la cultura, la clase social, el lugar geográfico, la orientación sexual, el cuerpo, entre otras. Así un hombre hegemónico es blanco, heterosexual, educado, de clase alta, aspecto físico cuidado... Estas intersecciones van conformando círculos de opresión donde según pertenezcamos (o no) a un grupo dominante ejerceremos opresión hacia los otros grupos. Ya Freire (1979) exponía la relación opresor/oprimido como la pérdida de identidad del otro/a y la desposesión de todo poder (cultural, individual, político...) del grupo oprimido en favor del dominante. Dar cabida a las diversidades (Sánchez, Melero y de la Rosa, 2016) en la educación es fundamental para cumplir con el derecho a la educación. Entendiendo éste como indisociable de los demás derechos (Tomasevski, 2004).

Siguiendo con este hilo argumentativo encontramos varias dimensiones de análisis que son fundamentales para nuestra investigación-acción: a) construcción hegemónica de masculinidad y feminidad a través de la socialización; b) intersección de diversas dimensiones de opresión (género, raza, clase...); c) concepción de la otredad, de la otra y del otro, por oposición y como dimensiones binarias y jerarquizadas (hombre vs mujer; heterosexual vs homosexual; blanco vs negro; rico vs pobre...); d) importancia de la educación para deconstruir las categorías dominantes de ser "hombre y mujer". Poner el acento en la importancia de la socialización de género hegemónica nos lleva a centrar la mirada en la educación que recibimos desde la infancia por diversos canales, entre los que la escuela y la educación formal ocupan una posición privilegiada en nuestra sociedad occidental. Es indiscutible, siguiendo a Dewey (1970), que la escuela guarda una relación importante en la socialización de niñas y niños, particularmente, contribuye a formar identidades, cuerpos, culturas, grupos sociales, emociones, conductas, etc.

En este sentido, la finalidad de este artículo es, por un lado, visibilizar la responsabilidad que las y los docentes tienen en la socialización y reproducción de desigualdades, particularmente, aquellas relacionadas con los géneros hegemónicos; y, por otro lado, proponer un proyecto de acción formativa del profesorado en su etapa inicial desde un enfoque feminista que des-patriarcalice y des-colonice la educación.

\section{Fundamentación teórica: Construcción de una pedagogía feminista para des-patriarcalizar y des- colonizar la formación inicial del profesorado}

En el caso de la educación de las mujeres y en el caso del reconocimiento de su derecho a la igualdad de oportunidades, en un aprendizaje donde se resalten sus capacidades y aportes, así como aquellas de sus realidades y necesidades que son diferentes de las de los hombres, y que algunos currículos vuelven explícito, los 
currículos ocultos reprograman estereotipos y tabúes acerca de lo que pueden y deben hacer hombres y mujeres. En las prácticas docentes se manifiestan en omisiones, períodos de atención, reforzamientos y otras distinciones, donde se estimulan la participación, liderazgo y aprendizaje de los hombres y la actitud pasiva de las mujeres. (Gargallo, 2008, p. 17)

Con estos cuestionamientos Gargallo (2008) nos invita a incluir una mirada feminista que de-construya los mandatos de género hetero-normativos expuestos en el apartado anterior. Siguiendo a la autora, el feminismo convierte la rabia ante la desigualdad en esperanza bajo el convencimiento de que las personas pueden reinventarse y reformularse mejor a sí mismas; y apuesta por desaprender las lecciones de género y la mirada masculina en lo cotidiano. Para contribuir a esta propuesta debemos poner el foco en la educación y la formación del profesorado como uno de esos mecanismos que utiliza el patriarcado para normalizar los citados estereotipos y mandatos de género.

Sánchez, Penna y de la Rosa (2016) afirman que educar desde el feminismo debe ser un acto amoroso de respeto a cada persona en sus diferencias. Lo que define esta educación es su sentido de lo humano y su entendimiento de las diversidades. Este paradigma parte de una dimensión cultural construida donde los genes solo determinan nuestros rasgos físicos, por el contrario, las personas nos construimos en un proceso de interacción dinámico y permanente como seres humanos diversos. Las anteriores autoras se preguntan si de verdad somos como somos. Si de verdad podemos decir que tenemos libertad para ser, sentir y vivir. Como hemos visto, los roles y los estereotipos de género ejercen una gran presión social ya que se consideran como lo normal y lo sano. Por ello ponemos el acento en

$$
\begin{aligned}
& \text { el cuerpo, en global, el cuerpo que viven, sienten, se relacionan entre sí. Cuerpos } \\
& \text { diversos, diferentes, altos, bajos, gordos, capaces, bellos, feos, cuerpos, en definitiva, } \\
& \text { que se posicionan en el mundo en un complejo entramado donde el contexto cultural, } \\
& \text { social, económico y moral juega un papel fundamental en el proceso multi- } \\
& \text { identitario de la persona. (Sánchez, Penna y de la Rosa, 2016, p. 41) }
\end{aligned}
$$

Clase social, etnia, sexo, género, identidades, afectos, cuerpos no normativos..., es decir, todo aquello que se sale de esos roles y estereotipos hegemónicos, se entremezclan en las personas conformando múltiples identidades para situarnos en un plano de interseccionalidades (Platero, 2012). Para desmontar estos mitos, estereotipos y prejuicios normativos la clave será preguntarnos cómo nos miramos a nosotras mismas y cómo miramos a aquellas personas que no cumplen con las expectativas culturales. En este proceso de-constructivo, ponemos el foco en una práctica de educación feminista con un marco cultural común, consensuado e integrador de las diferencias. En este sentido, las niñas y niños necesitan aprender a pensar, a hablar, a sentir y a actuar desde el respeto a las diferencias. Todo este proceso de enseñanza y aprendizaje (construcción de-construcción) tiene en la formación del profesorado un aliado poderoso.

Como ha quedado expresado en la introducción, el enfoque cultural atraviesa todo el artículo pretendiendo hacer de la formación del profesorado una herramienta más que contribuya a modular una pedagogía feminista crítica (Cabello Martínez y Martínez Martín, 2017; Martínez Martín, 2016) y de inclusión de las diversidades. Se trata de un proceso donde el diálogo es la vía de construcción de la diversidad al dar la posibilidad de poner en interacción las diferencias, desde lo enriquecedor y positivo del conocimiento, el respeto y la comprensión. Este artículo trata de hacer una propuesta pedagógica superando el concepto de normalización y haciendo de las diferencias parte esencial de los derechos humanos y de la ciudadanía. 
Clave para comprender esta propuesta es tener claro el concepto de diversidad y su relación con la pedagogía feminista. Entendemos que los seres humanos no aprendemos de una misma manera, por ello, un reto clave de la educación es dar cabida a las diferencias individuales y sociales desmontando esos estereotipos y prejuicios aprendidos.

\subsection{Pedagogía feminista y diversidades: pautas desde la des-patriarcalización y la des-colonización}

Hablar de una pedagogía feminista es hablar de interseccionalidad, de multidimensionalidad, de la inclusión de las diferencias y de transformación social. Es diálogo, compresión, colectividad, educación popular, empoderamiento y ruptura de los círculos de opresión. Tal y como venimos exponiendo hasta el momento el feminismo, asociado con la pedagogía, busca superar una educación tradicional y reproductora de desigualdades.

La educación será ante todo guiar a la persona en su marcha responsable a través del tiempo. Educar-le será despertarle o ayudarle a que se despierte a la realidad en modo tal que la realidad no sumerja su ser, el que le es propio, ni lo oprima, ni se derrumbe sobre él. (Zambrano, 1965, p. 11; citado en Blanco, 2006, p. 162)

Estas palabras de Zambrano nos remiten al pensamiento de Freire (1979) al señalar la educación como una práctica de libertad. En este sentido, la pedagogía feminista es una preparación para la práctica de la autonomía, la emancipación, la crítica y el empoderamiento. Al posicionarnos en una pedagogía feminista nos reconocemos personal y profesionalmente en un compromiso de ruptura con las narraciones androcéntricas y coloniales, donde se legitima una única manera (blanca, masculina, heterosexual) de saber y hacer incardinada en una racionalidad técnica y positivista.

Por el contrario, reconocer la existencia de una diversidad de saberes que incluyan lo subalterno implica tener en cuenta la existencia de múltiples sujetos experienciales, sean mujeres u hombres, blancos o negros... sin jerarquizar, ni dicotomizar sus conocimientos. Esta ruptura con el orden establecido es difícil y supone romper con la zona de confort (Korol, 2016). La pedagogía feminista recurre a paradigmas emancipadores y críticos que incluyan otras maneras de conocer desde lo colectivo y la creación de redes, como, por ejemplo: la inclusión de las emociones y los afectos, las metodologías de la educación popular (teatro foro, asambleas, lecturas, desarrollo local...), los lenguajes inclusivos y respetuosos, la acción para la transformación, entre otros aspectos.

Reconocer la diversidad de saberes, de experiencias, de formas de sentir... lleva implícito la necesidad de des-patriarcalizar y des-colonizar la educación y la formación del profesorado. Situarnos en un paradigma postcolonial hace referencia a la recuperación y valorización de los saberes subalternos. Mohanty (2008) y Jabardo (2010) argumentan que existe una brecha de conocimiento entre lo local y lo global, el Norte y el Sur, lo masculino y lo femenino, en relación al género. Defienden, en este sentido, la necesidad de unas pedagogías antipatriarcales, antiglobalizadoras y anticoloniales. Mohanty (2008) afirma que "quizás ya no se trate simplemente de los ojos de occidente sino de cómo occidente está dentro y reconfigura constantemente y globalmente los términos de raza y género" (p. 432)

Galindo (2014) y Paredes (2010), reconocen que la des-colonización y la despatriarcalización son matrices importantes de la lucha feminista post-colonial y comunitaria. Ambas autoras, al referirse a estos conceptos señalan que no puede 
entenderse uno sin el otro. Estas mismas autoras hacen referencia a la necesidad de rehabitar y re-significar saberes, cuerpos, identidades, ciudadanías, acciones... En este proceso la educación no puede permanecer neutral, por ello se hace imprescindible construir y visibilizar una pedagogía feminista que reconstruya desde lo popular, lo local, lo subalterno... las interpretaciones culturales que han sido obligadas a permanecer en los márgenes de las lógicas dominantes, proponiendo la necesidad de una de-construcción colonial del pensamiento y las acciones. Nos referimos, por lo tanto, a un feminismo postcolonial y de los Sures.

Para Paredes (2010) y Zabala (2012) resignificar la educación bajo las claves de la despatriarcalización y des-colonización implica: a) un feminismo comunitario como construcción de derechos individuales y colectivos y un desarrollo basado en el decrecimiento y la justicia social; b) dar voz y poder a las comunidades indígenas, campesinas, excluidas, negras...; c) incluir las diferencias y las diversidades a partir de creativas formas de comunicación (educación popular, técnicas como el teatro-foro, asambleas, música, simbología, libros, cuerpos...; d) creación de redes de sororidad entre mujeres que favorezcan la toma de conciencia individual y colectiva de las diversas opresiones y violencias; e) procesos de educación comunitaria y permanente para el empoderamiento.

En concordancia con estos planteamientos, siguiendo a Martínez Martín (2016), cabe destacar los principales rasgos definitorios para la construcción de una pedagogía feminista que des-patriarcalice y des-colonice la formación del profesorado:

- Partir de una educación política y feminista la cual debería impartir enseñanzas sobre una ciudadanía activa en las luchas por la justicia social.

- Incluir los saberes y experiencias de las mujeres rompiendo las dinámicas coloniales de construcción del conocimiento.

- Educar en la capacidad crítica, entendiendo que el mundo puede ser transformado y comprendido desde su diversidad.

- Reconocer y de-construir las relaciones entre poder y educación incluyendo en los currículos educativos los saberes diversos.

- Releer la realidad, nuestras prácticas, a partir del saber popular y la historia de las opresiones.

- Promover una dimensión dialógica que se fundamente en la importancia de la diversidad de narrativas, en las historias de vida, en las reflexiones compartidas y en la construcción colectiva de conocimiento.

- Asumir la dimensión grupal para promover un desaprendizaje colectivo de las opresiones.

- Apostar por la praxis, unión de teoría con la práctica, que permita transformar realidades individuales y sociales y acercarnos al conocimiento desde otras esferas más allá de la racionalidad técnica (colonizadora) apostando por un enfoque emancipador y crítico del conocimiento que incluya las experiencias, las emociones, los sentidos, las interpretaciones diversas de las realidades, entre otras.

Para construir esta pedagogía, poniendo en práctica los anteriores rasgos definitorios, se considera la formación del profesorado una herramienta fundamental de compromiso y 
acción, como se ha ido expresando a lo largo de todo el artículo. Se reconoce la formación inicial del profesorado como un momento prioritario para sensibilizar y visibilizar las desigualdades de género normalizadas a través de la educación; y para poner de manifiesto la necesidad de transformación de lo individual y lo colectivo. Consideramos imprescindible poner en valor los saberes del propio profesorado, sus vivencias y experiencias, sin imponer un enfoque de carencias sino tratando de dar valor a la construcción colectiva del conocimiento y el compromiso por el derecho a una educación de calidad para todas y todos. Sistematizando las propuestas de construcción de una pedagogía feminista, ésta tiene que ser capaz de apoyar procesos de empoderamiento, generar autonomía, libertad y emancipación entre las personas capaces de contribuir a generar una educación crítica y transformadora que sea garante de equidad y justicia social.

\section{Método}

Se toma como referencia una metodología de enfoque cualitativo y emancipador con carácter analítico-crítico y fundamentado en la práctica de la investigación-acciónparticipativa. El diseño de la investigación se ha dividido en las siguientes fases: 1) definición del problema a partir de un estudio documental y bibliográfico en torno a narrativas contra-hegemónicas feministas; 2) discusión teórica para la construcción de una pedagogía feminista postcolonial y su conexión con la formación del profesorado; y 3) puesta en práctica de un proyecto piloto de formación feminista del profesorado y su posterior evaluación y proyección.

En la primera y segunda fase de estudio documental y de discusión teórica se han tenido en cuenta los principios básicos de las epistemologías del Sur (Sousa, 2010) y contrahegemónicas para descolonizar los saberes y reinventar las dinámicas de poder. Siguiendo con estos principios los criterios de selección bibliográfica y documental han sido: a) la presencia de narrativas diversas dentro de una misma realidad (feminismos comunitarios, queer, de las diferencias...); b) la construcción de conocimiento desde los Sures (feminismos africanos, latinoamericanos y occidentales críticos); c) el rechazo a la universalización y generalización del conocimiento (enfoques cualitativos y reflexivos, emancipadores y postcoloniales); d) la relación objeto-sujeto desde el respeto a las subjetividades; y e) la interrelación de diversas fuentes de conocimiento y saber (feminismos, educación y formación del profesorado).

Siguiendo a Sousa (2010) tener en cuenta una epistemología de los Sures conecta con el saber subalterno de mujeres negras, campesinas, africanas y latinoamericanas con la finalidad de incluir las diversidades de narrativas existentes dentro de una misma realidad. Tanto el estudio documental y bibliográfico como la discusión teórica contribuyen a fundamentar el complejo estado de la cuestión desde tres ejes interrelacionados: la educación, la formación del profesorado y el feminismo. Teniendo la perspectiva postcolonial como eje transversal a todo el proceso marcando la diferencia en las formas de leer y escribir dichas realidades.

Entre las principales fuentes de información se tienen en cuenta las líneas de pensamiento generadas por las luchas feministas de las últimas décadas, en las cuales está tomando un protagonismo cada vez mayor la presencia de voces desde las fronteras. Se suman, además, diversas investigaciones realizadas en el marco de una Tesis Doctoral 
(Cabello Martínez y Martínez Martín, 2017) que sirven como referentes en el acceso a fuentes documentales y posicionamientos epistemológicos. Derivado del anterior proceso de discusión teórica se evidencian los mecanismos de reproducción de las desigualdades patriarcales y la responsabilidad que tiene la educación en ello. Como consecuencia se propone la construcción de una pedagogía feminista que dé respuesta a tales necesidades: des-colonizar y des-patriarcalizar la educación y la formación del profesorado. Esta propuesta teórica queda fundamentada en el citado proceso reflexivoanalítico, siendo éste el objetivo principal de la investigación.

\subsection{Participantes, técnicas de recogida y análisis de la información}

Asumiendo los principios de la investigación-acción-participativa (IAP), donde se busca la transformación de los contextos concretos, en la tercera fase de la investigación se pone en práctica un proyecto piloto de formación docente que contempla los principios de la citada pedagogía feminista para su posterior evaluación y mejora. Esta experiencia se ha llevado a cabo en los cursos de primero de Magisterio de Educación Infantil de la Universidad Complutense de Madrid, y en el grado de Educación Social de la Universidad de Castilla la Mancha durante los cursos 2015-2016 y 2016-2017. El total tomamos como muestra 120 estudiantes entre ambas universidades por curso.

Como principal técnica de recogida de datos dentro del proceso de la IAP se ha contado con un registro de observación participante a partir de una tabla de categorías validada a través de un juicio de expertas, destacando principalmente aquellas relacionadas con: a) identificación conceptos clave de igualdad y género; b) relación de dichos conceptos con las desigualdades cotidianas; c) identificación de aspectos educativos y la desigualdad de género; d) reproducción de estereotipos y creencias machistas.

Otra importante técnica utilizada ha sido un grupo de discusión en torno a los temas trabajos en las sesiones del taller (formación feminista del profesorado) ampliando y profundizando en la construcción colectiva de una pedagogía feminista transformadora. Además, para el análisis de la información recabada se ha contado con el apoyo visual de los materiales generados durante los procesos de reflexión y acción en los talleres, junto con el análisis de la tabla de categorías.

\section{Resultados: ¿Qué traemos en nuestras mochilas? Experiencias de formación feminista del profesorado}

Los roles y mandatos de género en los que somos socializadas desde la infancia contribuyen a modelar nuestra identidad: emociones, percepciones, relaciones intra e interpersonales, expectativas, deseos, etc. Al naturalizar los elementos de socialización legitimamos su perpetuación, de forma más o menos consciente. Una persona adulta proyecta y reproduce roles y mandatos con la intención de mostrar los modelos de feminidad y masculinidad válidos, a pesar de que las formas sean diferentes de las que aprendió en su momento. Algunos de estos modelos pueden incluso dar la impresión de una igualdad ya alcanzada, lo que Marcela Lagarde (2012) llama "el velo de la igualdad".

Se hace imprescindible reflexionar entonces sobre las prácticas cotidianas tanto a nivel personal como a nivel profesional, y sobre las ideas que subyacen a estas prácticas y que sostienen un sistema injusto y desigual. La escuela es un espacio excepcional para favorecer procesos de emancipación y transformación, pero sin una formación del 
profesorado crítica y reflexiva, existe el peligro de que se sigan perpetuando los mecanismos de reproducción del sistema hetero-patriarcal.

En este sentido, el sistema educativo puede ser a su vez un espacio de transformación, pero también de adoctrinamiento, reproducción y legitimación de mensajes de opresión. Tiene la pedagogía feminista una responsabilidad fundamental para evitar dicha reproducción y facilitar los procesos de reflexión-acción sobre nuestras prácticas e imaginarios cotidianos.

\subsection{Propuesta acción: proyecto piloto de formación inicial del profesorado}

Una vez presentados los principios y pautas como propuesta para una construcción de una pedagogía feminista y teniendo en cuenta el compromiso por la transformación de nuestros contextos cotidianos, proponemos una puesta en acción centrada en la formación inicial del profesorado. En esta propuesta de acción se aborda la formación inicial del profesorado en dos grandes fases: 1) sensibilización feminista sobre aquellos estereotipos y mandatos de género que aprendemos y que, como hemos visto, reproducimos en nuestro ejercicio profesional; y 2) construcción de nuevas formas de ejercer la docencia atendiendo a la construcción de materiales, contenidos, lenguajes, acciones, etc. desde un punto de vista feminista, descolonizador y crítico. A continuación, pasamos a exponer los principales resultados de cada una de las fases desarrolladas durante la experiencia de formación feminista del profesorado:

Sensibilizar acerca de la construcción social del patriarcado es complejo y exige, además de tiempo, un rigor científico apoyado por las teorías feministas.

En este proceso de análisis la reflexión se centra primeramente en visibilizar cómo son construidos los estereotipos, los prejuicios y los valores hegemónicos. Y, en segundo lugar, en entender que, cómo docentes, también somos socializadas en el mismo sistema y que a su vez transmitimos esos constructos sociales a través de la educación. Dicho de otro modo, se hace imprescindible de- construir todos aquellos imperativos y aprendizajes que traemos en nuestras mochilas y reproducimos como maestras y educadoras.

En esta primera fase el objetivo principal es identificar los mecanismos por los que interiorizamos y naturalizamos de manera sutil (o no tan sutil) roles y mandatos de género, en el que se sustenta un mundo dicotómico, jerarquizado y generizado. Se trabaja desde la propia experiencia tanto individual como colectiva: análisis de imaginarios en torno a la feminidad y masculinidad, expectativas, deseos, tipos de ocio, emociones, empleo de tiempos, etc. En este sentido se busca sensibilizar acerca de la construcción de feminidades y masculinidades hegemónicas que conforman un único tipo de ser mujer y ser hombre. Si no cumples con estas expectativas estás fuera de la norma y por lo tanto serás excluida y disciplinada.

Esta reflexión viene acompañada de una toma de conciencia acerca de los mecanismos de reproducción social que normalizan la cultura machista y hetero-patriarcal, haciendo especial hincapié en la labor del profesorado. En este sentido, atendiendo al análisis por categorías derivado de la observación participante y de los grupos de discusión, se detectan una serie de mecanismos de reproducción (los más repetidos) normalizados por las y los participantes: 
- Normalización de las estructuras jerarquizadas de dominación: hombre/mujer, norte/sur, rico/pobre... en la construcción de las identidades individuales pero también como parte de la estructura del sistema educativo.

- Invisibilización de las diferencias, con una mayor tendencia hacia la integración más que hacia la inclusión. Se detecta, además, que existe un discurso de las diversidades a nivel teórico que no tiene respuesta activa en la práctica cotidiana, por ejemplo, al tratar casos de transfobia u homofobia.

- Desconocimiento de conceptos clave ligados al pensamiento feminista como son: construcción sexo/género, interseccionalidad de exclusiones, diversidad sexual, violencias de género, entre otras.

- Se detectan conductas machistas generales, como puede ser en cuentos, películas, canciones, acoso... pero pasan desapercibidas las conductas machistas cotidianas y normalizadas en nuestro día a día, como por ejemplo: la división del trabajo, de los tiempos, la feminización de ciertas ocupaciones, entre otras.

- Existe una preocupación generalizada por los casos de violencia de género, pero se desconocen los procesos, los datos reales, se cae en falsas creencias y no se aprecia una posición clara de pautas de actuación desde su futura laboral como docentes.

- Es compartida la opinión de que la pedagogía feminista es necesaria para actuar sobre las desigualdades de género, pero reconocen el desconocimiento hacia este tema y cómo poder aplicarlo a su práctica docente cotidiana más allá de días especiales o semanas concretas.

No atender a esta fase de reflexión y visibilización provoca que reproduzcamos en el aula o en el contexto educativo actitudes sexistas, homófobas, racistas, transfóbicas... Y, en definitiva, que no atendamos a las diversidades y diferencias no cumpliendo con un derecho a la educación de calidad para todas y todos. Es imprescindible que desde la formación inicial del profesorado las personas que van a enfrentarse a procesos de acompañamiento, educación y apoyo tomen conciencia de su agencia como elementos de socialización y de la carga que esto implica.

Construir nuevas formas feministas de llevar a cabo los procesos de enseñanza y aprendizaje nos lleva a tener en cuenta varios aspectos clave: selección de contenido, uso de lenguaje inclusivo, distribución de espacios y tiempos, propuesta de actividades y juegos, actitudes ante las diferencias, entre otras.

En esta segunda parte del proceso formativo se busca subvertir las prácticas docentes transformado aquellos elementos de reproducción patriarcal. Así, se trabaja sobre los aspectos básicos del currículo educativo de cada etapa. Por ejemplo, se incluye una perspectiva feminista en la construcción de identidades, la ocupación del espacio, los cuentos y literatura infantil, los juegos y juguetes, el lenguaje. En la figura 1 se reflejan algunos ejemplos de lo dicho hasta el momento.

Bajo la consigna "reproducción vs transformación" se busca visibilizar por un lado aquellos mecanismos de socialización patriarcal que aparecen en las diferentes dimensiones propuestas para el análisis (recreo, cuentos, cuerpos, lenguaje). Y por otro lado se pretende iniciar un proceso de subversión dando pautas y claves para el cambio 
en su futura labor docente. Para esta fase partimos de las vivencias de cada persona, buscando conectar su propia experiencia con la fase previa de identificación. Nos parece importante confrontar los procesos de "reproducción vs transformación" para apoyar el mensaje de que existen posibilidades de cambio reales y que se puede empezar en ese mismo momento, a partir de una reflexión individual enriquecida con las propuestas del grupo. En este sentido, creemos en el trabajo colectivo como herramienta para un cambio radical y significativo.

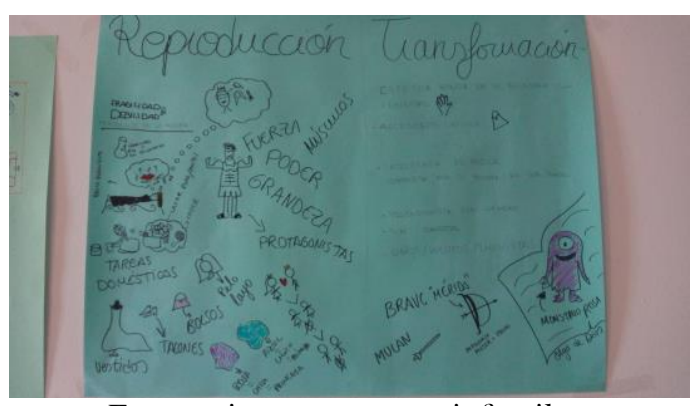

Estereotipos en cuentos infantiles

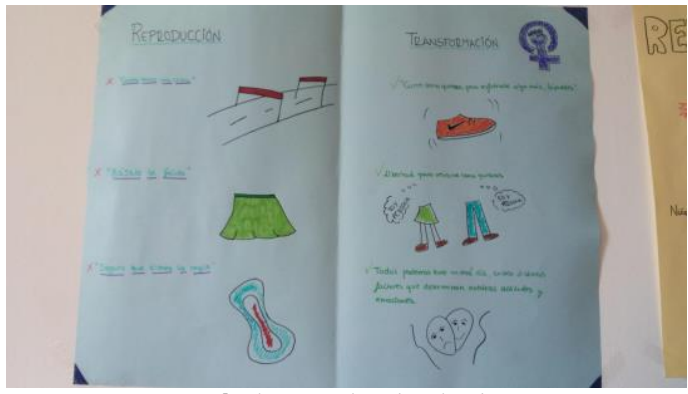

Uso de lenguajes inclusivos

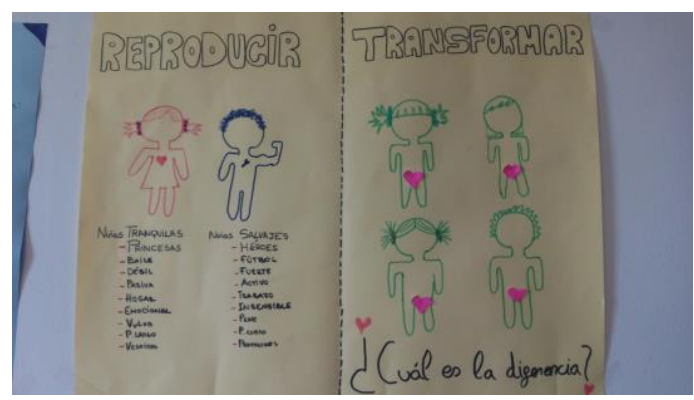

Diversidades identitarias

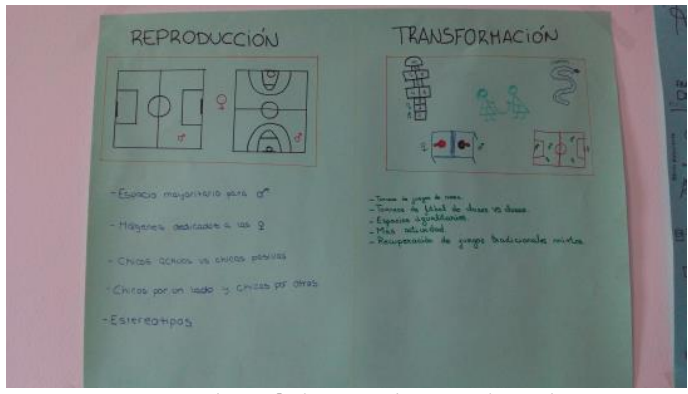

Ocupación del espacio en el patio

Figura 1. Materiales construidos en el aula

Fuente: Elaboración propia a partir de material visual de la experiencia formativa.

Este trabajo de acción y transformación es completado con otras propuestas como, por ejemplo, la construcción de materiales didácticos feministas (juegos, cuentos, canciones...) y de unidades didácticas que tengan en cuenta las diferencias, el conocimiento de materiales feministas ya existentes, elaboración de un banco de recursos y buenas prácticas, la invitación a continuar con su formación permanente, entre otras. Cabe recordar que esta experiencia se centra en la formación inicial del profesorado y que por lo tanto no estamos ante profesionales en ejercicio, el objetivo principal es reflexionar y aprender nuevas formas de hacer educación para su futura proyección. Es igualmente importante reseñar que hasta la fecha de publicación de este artículo estamos ante un proyecto piloto que no cuenta con financiación externa por lo que los espacios y tiempos dedicados a la experiencia se vinculan al interés de las autoras.

\section{Discusión y conclusiones}

La naturaleza de permanente cuestionamiento del saber hegemónico supone un compromiso con la lucha feminista en relación a la de-construcción de los imperativos hetero-patriarcales y una importante propuesta de acción socio-educativa; pero también es causante de dificultades que, aunque no es posible relatar de manera exhaustiva, 
trataremos de presentar en formato de retos para seguir indagando en el final de este apartado, tal y como se han ido viviendo y sintiendo.

Primeramente, hacemos referencia a los principales resultados derivados del proceso de transformación docente, a saber:

- identificación y diagnóstico: la necesidad de posicionarse en un plano de pensamiento y acción feminista postcolonial ante la reproducción de desigualdades de género y a través del ejercicio de la docencia;

- propuesta: construcción de una pedagogía feminista para la formación del profesorado bajo los principios de transformación y crítica social para descolonizar y des-patriarcalizar saberes y prácticas educativas;

- acción-evaluación: puesta en práctica de una experiencia piloto para subvertir la formación inicial del profesorado en temas de género e igualdad.

En este proyecto de transformación docente las teorías y prácticas feministas se presentan como alternativa para incluir las diferencias y aquellas narrativas que permanecen en los márgenes de lo dominante y construir, así, desde lo subalterno una educación que supere la reproducción sistemática de desigualdades.

En segundo lugar, llamamos la atención sobre las aportaciones científicas de este artículo que: permiten crear conocimiento a partir de la reflexión crítica para una despatriarcalización y des-colonización de los saberes y prácticas dominantes en la formación inicial del profesorado (Galindo, 2013). Al tratarse de una propuesta de formación inicial del profesorado se busca la proyección futura de las prácticas educadoras teniendo como objetivo transversalizar la pedagogía feminista haciendo incidencia en los contenidos, lenguajes, metodologías, evaluaciones...y, en definitiva, en los currículos y proyectos educativos (Sánchez, 2014).

En tercer lugar, como sistematización de la discusión pedagógica-feminista (Amorós, 2007) llevada a cabo, destacamos:

- La normalización del sistema patriarcal cuenta con el apoyo político no habiendo una voluntad clara para subvertir las situaciones de desigualdad, prueba de ello son la falta de instituciones y proyectos formativos para el profesorado con carácter feminista.

- Ante la falta de crítica y reflexión sobre los agentes de socialización patriarcal surge la necesidad de hacer un trabajo previo de reflexión individual para identificar situaciones personales de opresión y privilegios. Este aspecto compete tanto al profesorado universitario o a formadores/as de formadores/as como al propio profesorado en formación inicial. No es posible desarrollar una pedagogía feminista sino se hace investigación y ciencia feminista.

- Para des-colonizar y des-patriarcalizar los saberes y conocimientos es necesario reivindicar los feminismos desde las fronteras, de los Sures y subalternos en la construcción de una pedagogía emancipadora y crítica que rompa con los círculos de opresión y colonización.

- Entender las diversidades como aspectos positivos que suman y crean ciudadanías basadas en la equidad y la justicia social. Aspecto fundamental para 
de-construir los cuerpos, identidades, sexualidades... normativos y empezar a educar en las diferencias no como la "excepción" sino como lo enriquecedor.

Retomamos las palabras de Ngozi (2013) como aviso sobre el peligro de conocer y crear conocimiento desde posicionamientos estáticos y cerrados, es decir, desde una sola lógica, que suele ser la dominante. En este artículo hemos tenido como base de estudio voces de mujeres y niñas más allá de las fronteras occidentales, y nos hemos hecho eco de las teorías que reclaman una des-colonización y des-patriarcalizarción de los imaginarios patriarcales. El proceso de fusionar ambos tipos de saberes se ha revelado como dinámico, diverso y comprensivo con las diferencias; es decir, como una integración de historias y de narrativas que van conformando las epistemologías de los "Sures". Este proceso, además, ha generado vivencias de dificultades, ha planteado nuevos retos y ha abierto líneas futuras de investigación para seguir indagando en estas temáticas.

Para finalizar con esta discusión y propuesta feminista planteamos una serie de retos de proyección futura que nos permita seguir avanzando en el camino de la visibilización y construcción de una pedagogía feminista crítica:

- Valoración y fomento de diversidades múltiples y cambiantes como una cuestión de derechos humanos fundamentales (y no sólo atención e inclusión de la diversidad)

- Introducción de la ética de cuidados como eje vertebrador de la práctica educativa

- Inclusión y desarrollo de pedagogías queer, y subversión de cuerpos normativos.

- Fomento de la formación del profesorado en un espacio de diálogo permanente entre la academia y la acción social.

- Desarrollo de espacios de debate y construcción colectiva dentro de la formación inicial del profesorado.

- Inclusión de metodologías formativas que fomenten la construcción colectiva del conocimiento: teatro foro, asambleas, lecturas, educación popular...

- Apuesta por la incidencia política respecto al diseño de planes de estudio desde una perspectiva feminista.

- Construcción de una pedagogía feminista que permita sentar las bases de una educación libre de violencias machistas y como práctica de libertades.

- Ocupación de espacios formativos más allá de los oficiales que permita introducir en la agenda educativa aspectos clave del movimiento feminista.

- Creación de redes de apoyo colectivo que permitan el empoderamiento y la creación de espacios comunes de ruptura de las opresiones, convirtiéndose en espacios de formación y construcción de conocimiento local.

- Avance hacia una racionalidad emancipatoria y crítica que des-colonice y despatriarcalice los conocimientos incluyendo procesos educativos que tengan en cuenta: las emociones, la afectividad, las intuiciones, las propias interpretaciones, los aspectos lúdicos, la variedad de cuerpos, la educación popular, el arte, el teatro del/las oprimidas, la expresión corporal, el diálogo... 
- Fomento del diálogo entre realidades, contextos y tiempos más allá de los tradicionales-escolares.

Desde el feminismo la consigna "lo personal es político" toma una relevancia importante al traspasar la frontera de lo cotidiano para hacer los problemas de las mujeres problemas sociales. Como tal la formación del profesorado exige una transformación en lo personal para llegar a una transformación de las prácticas, tenemos responsabilidad como ciudadanas y ciudadanos críticas, pero también como formadoras de futuro en el camino hacia la justicia social y la equidad.

$$
\begin{array}{r}
\text { Quisiera entrar por el ojo de una aguja } \\
\text { Al reino de la gente } \\
\text { Donde ninguna edad sea pecado } \\
\text { Ningún sexo demasiado pequeño } \\
\text { Ningún ser un poco menos. } \\
\text { (Laura Devetach, 2009) }
\end{array}
$$

\section{Referencias}

Amorós, C. (2007). Teoría feminista: De la ilustración a la globalización. Madrid: Minerva Ediciones.

Beauvoir, S. (1981). El segundo sexo. Buenos Aires: Siglo Veinte.

Blanco, N. (2006). Saber para vivir. En A. M. Piussi y A. Mañeru (Coords.), Educación, nombre común femenino (pp. 158-183). Barcelona: Octaedro.

Butler, J. (2007). El género en disputa: Feminismo y la subversión de la identidad. Madrid: Paidós.

Cabello Martínez, M. J. y Martínez Martín, I. (2017). Aportes teóricos de la perspectiva de género en la mejora de la educación de las niñas en África. Educación XX1, 20(1), 163-181.

Connell, R. W. (1997). La organización social de la masculinidad. Isis Internacional Ediciones de las Mujeres, 24, 31-48.

Dewey, J. (1970). Democracia y educación. Buenos Aires: Losada

Freire, P. (1979). La educación como práctica de libertad. Madrid: Siglo XXI.

Galindo, M. (2013). No se puede descolonizar sin despatriarcalizar. Sucre: Mujeres Creando.

Galindo, M. (2014). A despatriarcalizar. Madrid: Traficantes de Sueños.

Gargallo, F. (2008). El feminismo y la educación en y para nuestra América. Revista Venezolana de Estudios de la Mujer, 13(31), 17-26.

Jabardo, M. (2010). Feminismos negros. Una antología. Madrid: Traficantes de Sueños.

Korol, C. (2016). Feminismos populares. Pedagogías y políticas. Buenos Aires: El Colectivo.

Lagarde, M. (2012). El feminismo en mi vida. Hitos, claves y utopía. Ciudad de México: Instituto de la Mujer.

Martínez Martín, I. (2016). Construcción de una pedagogía feminista para una ciudadanía transformadora y contra-hegemónica. Foro de Educación, 14(20), 129-151. https://doi.org/10.14516/fde.2016.014.020.008

Mohanty, C. (2008). Bajo los ojos de occidente: academia feminista y discursos coloniales. En L. Suarez y A. Hernández (Eds.), Descolonizando el feminismo: teorías y prácticas desde los márgenes (pp. 117-164). Madrid: Cátedra. 
Ngozi, C. (2013). El peligro de una sola historia. Recuperado de http://www.youtube.com/watch?feature=player_embedded\&v=4gH5oB1CMYM\#t=1 1

Ngozi, C. (2015). Todos deberíamos ser feministas. Madrid: Penguin Random House.

Paredes, J. (2010). Hilando fino desde el feminismo comunitario. La Paz: Mujeres creando comunidad.

Platero, L. (2012). Intersecciones: cuerpos y sexualidades en la encrucijada. Barcelona: Bellaterra.

Sánchez, M. (2014). Género, diversidades y diferencias en educación infantil y primaria. Madrid: Fundación $1^{\circ}$ de Mayo.

Sánchez, M., Penna, M. y de la Rosa, B. (2016). Somos como somos. Deconstruyendo y transformando la escuela. Madrid: La Catarata.

Segato, R. (2016). La guerra contra las mujeres. Madrid: Traficantes de Sueños.

Sousa, B. (2010). Descolonizar el saber, reinventar el poder. Montevideo: Trilce.

Tomasevski, K. (2004). El asalto a la educación. Madrid: Intermón Oxfran.

Varela, N. (2008). Feminismo para principiantes. Barcelona: Ediciones B.

Zabala, L. (2012). Descolonizar la descolonización desde los feminismos. En C. Sánchez (Comp.), Mujeres en diálogo: Avanzando hacia la despatriarcalización en Bolivia (pp.165-180). La Paz: Coordinadora de la Mujer.

\section{Breve CV de las autoras}

\section{Irene Martínez Martín}

Doctora Cum Laude en Educación, mención doctorado europeo. Profesora Asociada en la Universidad de Castilla la Mancha y la Universidad Complutense de Madrid en los Grados de Educación Social, Magisterio y Pedagogía desde el curso 2015 hasta la actualidad. Además de dirigir TFG y TFM de las anteriores titulaciones. Principales líneas de investigación vinculadas a los feminismos postcoloniales y de las diversidades, a la pedagogía feminista y crítica y la educación y el desarrollo en África. Bajo estas líneas he publicado artículos científicos y participado en foros de debate de interés para el área, tanto nacionales como internacionales. Además de ser ponente en cursos y seminarios de formación acerca de las Pedagogías Feministas. ORCID ID: 0000-00019131-9057. Email: imartio2@ucm.es

\section{Gema Ramírez Artiaga}

Educadora social, licenciada en pedagogía y master en estudios interdisciplinares de género. Desde 2014 inmersa en proyectos de formación y sensibilización en igualdad de género en centros educativos y universitarios. Colaboradora con la Universidad Complutense de Madrid y la Universidad de Castilla La Mancha en proyecto de formación inicial y sensibilización en los grados de Educación Social, Pedagogía y Magisterio. Participa en varios colectivos y plataformas feministas. Desde 2015 trabaja como educadora en un recurso específico para mujeres víctimas de trata y explotación sexual. ORCID ID: 0000-0002-7323-5471. Email: iramirez.gema@gmail.com 